\title{
THE ELASTIC-PLASTIC CYLINDER SUBJECTED TO RADIALLY DISTRIBUTED HEAT SOURCE, LATERAL PRESSURE AND AXIAL FORCE WITH APPLICATION TO NUCLEAR REACTOR FUEL ELEMENTS*
}

\author{
By T. B. Kammash†, S. A. Murch $\ddagger$ and P. M. Naghdi
}

(Received 20 July 1959)

\begin{abstract}
SUMMARY
THE elastic-plastic deformation of a solid cylinder in the presence of a distributed heat source and subjected to a lateral pressure $p$ and an axial fource $F$ is considered in detail using Tresca's yield function, its associated flow law, and a linear workhardening law. Particular attention is given to the class of problems in which the radial stress. and not the axial stress, is the intermediate principal stress. The general results are applied to the cylindrical nuclear reactor fuel element in the state of plane strain with a radially distributed Gaussian heat source, acting in its fissionable interior. The solution is expressed in closed form in terms of the exponential integral and the incomplete gamma function and is found to exhibit three stages of plastic deformation. In stage I the plastic domain consists of two concentric and adjacent zones with different stress fields and different rates of propagation which expand outward from the centre of the cylinder ; in stage II the elastic domain vanishes, while the inner plastic zone continues to propagate ; and in stage III a third plastic zone is formed at the outer radius which propagates inward. Throughout the deformation the states of stress are found to exhibit regular progression. A numerical example is included.
\end{abstract}

\section{InTRODUCTION}

ThE brief list of existing complete solutions to elastic-plastic problems in the incremental strain theory of plasticity has been enriched in recent years by a number of thermoelastic-plastic investigations, e.g., Pakkus (1954), BLand (1956) Weiner (1956), Wilhoit (1958) and Cowper (1958). Of the problems solved in the above collection, all but the first and third employ Tresca's yield condition ; and of the plane strain solutions all contain, as do the isothermal solutions obtained to date, the axial stress $\sigma_{z}$ as the intermediate principal stress. Furthermore, in all cases the state of stress is statically determinate.

An exception may be found in the recent work of Weiner and Huddeeston (1959) who have obtained, for an incompressible nonhardening cylinder in the presence of a temperature gradient, a radially symmetric solution for the stress rates which for certain regimes of the Tresca yield surface is necessarily statically indeterminate.

\footnotetext{
-The results presented in this paper were obtained in the course of research sponsored by the Office of Ordnance Reserrch, U.S. Army. The investigation was initiated under Contract DA-20-018-ORD-14447 with the University of Michigan, and was completed under Contract DA-01-200-ORD-171 with the University of California at Berkeley.

+ University of Michigan, Ann Arbor, Michigan.

$\downarrow$ University of California, Berkeley, California.
} 
Among the existing radially symmetric solutions associated with the problem of the elastic -plastic cylinder. that of the thick-walled tube has been the subject of numerous investigations. Of particular interest is the work of Korrek (1953) who obtained a simple and satisfactory solution in closed form for a nonhardening compressible elastic-plastic material, using 'Trese'a's yield condition and its associated llow rule. 'This was extended by BIAND (1956) to include workhardening and a steady state temperature gradient. In both of these investigations (a) $\sigma_{z}$ is the intermediate principal stress so that. on account of the symmetry of the problem, the stress field is statically determinate, (b) no singular regime of the Tresea loading function is concountered and (c) the initial yield begins at the mner surface. developing a plastic region with a surrounding clastic annulus.

'The problem considered here is that of the quasi-static deformation of an clastic-plastic eylunder, which, with referenee to 'ylindrical polar coordinates $(r, \theta, z)$ oceupies the region $0 \leq r, a$ and is subjerted to a lateral pressure $p$, an axial foree $F$ and a radially distributed heat source $(Q$. all of which increase monotonically with time $t$. 'The solution is obtained for an inviseid workhardening material, with 'Tresca's loading function, its associated flow rule, and a workhardening law based on the plastic work per unit volume expended; the nonhardening elastic plastic solution is also deduced as a special case for comparison purposes. Depending upon the relative ralues of the mdependently rarying load parancters.' $p, Q$ and $F$, the initial yeld may occur at any radius $0=r=a$, while the axial component of stress $\sigma_{z}$ may or may not be the intermediate principal stress. Indecd, in the class of problems considered, the initial yicld oceurs along the axis of the cylinder $r$ - o, when the state of stress reaches the singular regime $\sigma_{\theta}-\sigma_{z}=\sigma_{r}-\sigma_{z}=2 k_{0}$. where $\sigma_{r}$ and $\sigma_{\theta}$ are respectively the radial and tangential components of stress, and $k_{0}$ is the yicld limit in simple shear. 'The ensuing state then consists of an clastic annulus $(\rho \leqslant r \leqslant a)$ surrounding a plastice region $(0 \leqslant r \leqslant \rho)$, with the clastic plastic boundary $r=\rho$. 'The solution in the plastic domain $(0 \leqslant r \leqslant \rho)$ does not lend itself to description by a single stress field; unstead, contmuity requirements, inposed on the stresses and displacement, at the clastic-plastic boundary $\rho$, together with consideration of the elastic loading paths as well as the possible paths that the stress might follow in the plastic range demand that the plastic domain $(0 \leqslant r \leqslant \rho)$ be composed of two concentric zones, separated by an interface $r \quad-\rho_{1}<\rho$, each with different stress fields and different rates of propagation. In this manner the state of stress is found to exhibit regular progression (following the terminology of Honge 1956) ; i.e., the state of stress upon yielding first lies on a regular face of the yield surface and then moves toward a singular regime where it stays throughout the remainder of the plastic deformation.

An interesting feature of the problem is the continued propagation of the interface boundary $\rho_{1}$, after the elastic plastic boundary $\rho$ has reached the outer surface $r--a$, so that the cylinder may sustain further loading. A second stage of the problem (referred to as Stage II) is thus entered in which the elastic region has vanished entirely. Stage II of the problem is short-lived, however, as the state of stress at $r=a$ reaches the regime $B$ before the boundary $\rho_{1}$ has moved appreciably (Fig. 1). and a third and final stage of the problem (Stage III) is entered. In Stage III the state of stress again exhibits regular progression, giving rise to a new zone $\rho_{2} \leqslant r=a$ (in which the state of stress corresponds to regime $\boldsymbol{B}$ ), initiating at 
$r=a$ with the interface boundary $\rho_{2}$ propagating inward; hence the two interfaces $\rho_{2}$ and $\rho_{1}$ approach each other. It is found that these boundaries never meet but approach limit values as the temperature tends to infinity. This observation is similar to that made by WIIIIOIT (1958) for a ring subjected to a temperature gradient, and the work of COWPER (1959) for a hollow sphere subjected to a temperature gradient.

Specifically, the contents of the paper are arranged in two parts. In Part I (Sections 1-4) of the present paper, following some preliminaries, the character of the initial yield for a given class of temperature distributions is discussed in detail. The differential equations for the stress fields corresponding to various regimes of the Tresca yield surface are derived for an arbitrary, radially symmetric, temperature distribution and without the specifications of any particular hardening law. Also included, is the solution for the case of a nonhardening elastic-plastic material.

As an application of the foregoing results in Part II (Sections 5-7), cylindrical nuclear reactor fuel elements in the state of plane strain are considered in detail. The solid cylinder of fissionable material forms the heat generating portion of the fuel element and is surrounded by a hollow cylinder of nonfissionable material called 'cladding.' The uniform pressure between the two cylinders is then a consequence solely of the temperature ficld generated (precluding a shrink fit for simplicity). Using a Gaussian heat source and a linear hardening law, the gencral differential equations for stress and the displacement derived in Part I, corresponding to the regimes which arise in the various stages of the problem, are solved and applied in the plastic regime. A numerical example is worked out and complete results presented for a workhardening material as well as a nonhardening material for comparison. A further comparison is made of the solution of the coupled mechanical-thermal problem considered here with that obtained by a simple superposition of solutions to the separate mechanical and thermal problems.

\section{PAR'I I. GENERAL CONSIDERATIONS}

\section{Preliminary Bickground}

With reference to cylindrical polar coordinates $(r, \theta, z)$, we recall for future reference that for axisymmetric problems in the state of plane strain the stress differential equations of equilibrium in the absence of body forces become simply

$$
\frac{\partial \sigma_{r}}{\partial r}+\frac{\sigma_{r}-\sigma_{\theta}}{r}=\mathbf{0},
$$

and the nonvanishing plane components of strain are given by

$$
\epsilon_{r}=\frac{\partial u}{\partial r}, \quad \epsilon_{\theta}=\frac{u}{r},
$$

where $u$ is the radial displacement. The elastic components of strain $\epsilon_{r}{ }^{\prime}, \epsilon_{\theta}{ }^{\prime}, \epsilon_{z}{ }^{\prime}$ are related to the stresses through the generalized Hooke's law, while the plastic components of strain $\left(\epsilon_{r}^{\prime \prime}=\epsilon_{r}-\epsilon_{r}{ }^{\prime}\right.$, etc) are expressed for workhardening materials by

$$
\left\{\dot{\boldsymbol{\epsilon}}_{r}^{\prime \prime}, \dot{\boldsymbol{\epsilon}}_{\theta}^{\prime \prime}, \dot{\boldsymbol{\epsilon}}_{\mathrm{z}}^{\prime \prime}\right\}=h\left\{\frac{\partial f}{\partial \sigma_{\tau}}, \frac{\partial f}{\partial \sigma_{\theta}}, \frac{\partial f}{\partial \sigma_{z}}\right\} \dot{f},
$$


where the plastic potential $f$ is regular, dot denotes differentiation with respect to time, and $h$ is a positive function (HrLl 1950). When the hardening is zero the quantity $h \dot{f}$ on the right-hand side of $(\mathbf{2 . 3})$ is to be replaced by $\dot{\lambda}$.

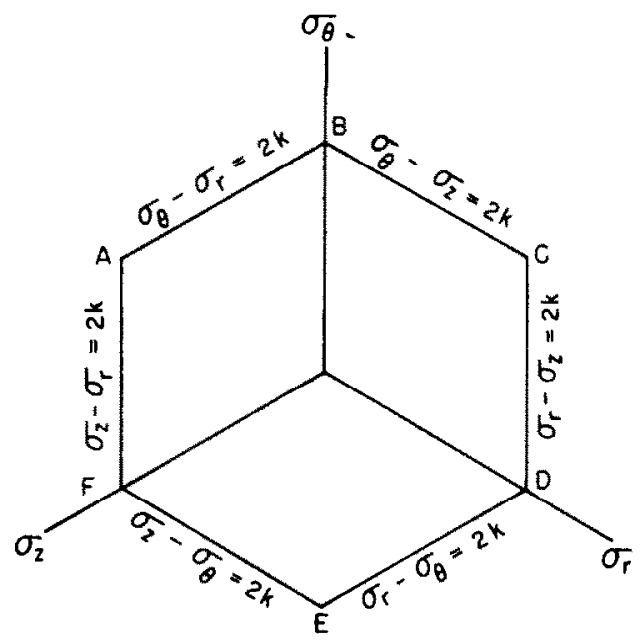

FIG. 1. Tresea's yield hexagon when $\sigma_{r}, \sigma_{\delta}, \sigma_{z}$ are the principal stresses.

Throughout this paper the plastic potential is taken as 'Tresca's yield function

$$
f=\max \left[\left|\sigma_{r}-\sigma_{\theta}\right|, \quad\left|\sigma_{\theta}-\sigma_{z}\right|, \quad\left|\sigma_{z}-\sigma_{r}\right|\right]=2 k,
$$

keeping in mind that the flow rule in the form (2.3) is valid only at regular regimes of (2.4); the function $f$ is shown schematically in Fig. 1 with the various regimes labelled. The material is assumed to harden according to the law

$$
2 \dot{k}=\eta W_{p}
$$

where $k$ is the yield stress in simple shear, $\eta$ is a constant which depends on the material, and

$$
W_{p}=\sigma_{r} \dot{\epsilon}_{\tau}{ }^{\prime \prime}+\sigma_{\theta} \dot{\epsilon}_{\theta}{ }^{\prime \prime}+\sigma_{z} \dot{\epsilon}_{z}^{\prime \prime}
$$

is the rate of plastic work per unit volume.

Although more general constitutive equations for non-isothermal plasticity have been recently proposed by $\mathrm{P}_{\mathrm{RAGER}}$ (1958), the thermal effect is here accounted for only in the generalized Hooke's law, so that the condition of compressibjity is

$$
\epsilon_{r}+\epsilon_{\theta}+\epsilon_{z}=\frac{1-2 \nu}{E}\left(\sigma_{r}+\sigma_{\theta}+\sigma_{z}\right)+3 x T,
$$

where $T$ is the change in temperature. The mechanical and thermal parameters $E, \nu$ and $\alpha$ (modulus of elasticity, Poisson's ratio and the coefficient of linear expansion, respectively), as well as the initial yield limit $k_{0}$, are assumed independent of temperature.

Since, for the problem being considered, the deformation is a direct consequence of the change in temperature arising from a distributed heat source within the body, any temperature difference that might occur due to the deformation itself 
The elastic-plastic cylinder subjected to radially distributed heat source

(as in thermoelastic damping) may be ignored, thus uncoupling the thermal and mechanical problems; furthermore, if the loading (thermal as well as mechanical) occurs quasi-statically, the heat conduction equation is characterized (e.g., THOMPSON and Rogers 1956, p. 165) by

$$
K \nabla^{2} T=-Q
$$

where $K$ is the thermal conductivity and $Q$ is a radially distributed thermal source expressible in terms of units of heat per unit time per unit volume, and

$$
\nabla^{2} \equiv \frac{\partial^{2}}{\partial r^{2}}+\frac{1}{r} \frac{\partial}{\partial r}
$$

\section{Tiffermofiastic Soletion and Initial. Yield}

While our main concern is with the elastic-plastic cylinder occupying the region $0 \leqq r \leqq a$, for future reference it is necessary to consider the corresponding thermoelistic problem. Specifically, we recall the (quasi-static) thermoelastic solution to the axisymmetric problem of a hollow cylinder $(\rho \leqq r \leqq a)$ in the state of plane stratin and subjected to a temperature distribution $T$, superposed on the elastic solution due only to a uniform a vial stress $\sigma_{z}=\mathrm{C}_{3}$ (e.g.. Tinoshrinko and GoODIER 1951, p. 409):

$$
\left.\begin{array}{rl}
u & =\frac{1+v}{1-v} \frac{\alpha}{r} \int_{\rho}^{r} \operatorname{Tr} d r+C_{1} r+\frac{C_{2}}{r}-\frac{v}{E} C_{3} r, \\
\sigma_{r} & =-\frac{\alpha E}{1-\nu} \frac{1}{r^{2}} \int_{\rho}^{r} \operatorname{Tr} d r+\frac{E}{1+\nu}\left(\frac{C_{1}}{1-2 v}-\frac{C_{2}}{r^{2}}\right), \\
\sigma_{\theta} & =\frac{\alpha E}{1-\nu} \frac{1}{r^{2}} \int_{\rho}^{r} \operatorname{Tr} d r-\frac{\alpha E T}{1-\nu}+\frac{E}{1+\nu}\left(\frac{C_{1}}{1-2 \nu}+\frac{C_{2}}{r^{2}}\right), \\
\sigma_{z} & =-\frac{\alpha E T}{1-\nu}+\frac{2 \nu C_{1} E}{(1-2 \nu)(1+\nu)}+C_{3}, \\
C_{3} & =\frac{-2 \nu}{1-2 \nu} \frac{C_{1} E}{1+\nu}+\frac{2}{a^{2}-\rho^{2}} \frac{\alpha E}{1-\nu} \int_{\rho}^{a} \operatorname{Tr} d r+\frac{F}{\pi\left(a^{2}-\rho^{2}\right)}
\end{array}\right\}
$$

where

The thermoelastic solution for the solid eylinder $(0 \leqslant r \leqslant a)$ may be deduced from (a.1) by allowing in the integrals the lower limit $\rho \rightarrow 0$, and by setting the coefficient $C_{2}=0$, which ensures the regularity of the solution at $r=0$. When this solution is subjected to the boundary conditions appropriate to the solid cylinder subjected to a radial temperature distribution $T$, a lateral pressure $p$, and an axial force $F$ (which includes the effects of both $C_{3}$ as well as that arising from the plane strain solution) the stresses are found to be

where

$$
\begin{gathered}
\sigma_{r}=\frac{\alpha E}{1-\nu}[\theta(a)-\theta(r)]-p . \\
\sigma_{\theta}=\frac{\alpha E}{1-\nu}[\theta(a)+\theta(r)-T]-p . \\
\sigma_{z}=\frac{\alpha E}{1-\nu}[-T+2 \theta(a)]+\frac{F}{\pi \iota^{2}}, \\
\theta(r)=\frac{1}{r^{2}} \int_{0}^{r} T(r) r d r .
\end{gathered}
$$


According to Tresea's yield condition yielding occurs when one of the quantities

$$
\begin{aligned}
& \left|\sigma_{r}-\sigma_{\theta}\right|=\left|\begin{array}{c}
\alpha \boldsymbol{E} \\
1-v
\end{array}[\boldsymbol{T}-\theta(r)]\right|, \\
& \left.\begin{array}{l}
\left|\sigma_{r}-\sigma_{z}\right|=\left|\begin{array}{c}
\alpha E \\
1-\nu
\end{array}[T-\theta(r)-\theta(a)]-\frac{F}{\pi a^{2}}-p\right|, \\
\left|\sigma_{\theta}-\sigma_{z}\right|=\left|\frac{\alpha E}{1-\nu}[\theta(r)-\theta(a)]-\frac{F}{\pi a^{2}}-p\right|,
\end{array}\right\}
\end{aligned}
$$

reaches the value $2 k_{0}$. Since the determination of the maximum of (3.3) depends on the load parameters and especially on the distribution of $\boldsymbol{T}$ (which is so far arbitrary), we restrict ourselves to the class of monotone temperature distributions which assumes its maximum either at $r=0$ or at $r=a$. With the notation

$$
\begin{aligned}
& \boldsymbol{H}=-\frac{\boldsymbol{F}}{\pi \boldsymbol{t}^{2}}-p, \quad m=\frac{v_{1}}{v_{2}}-1, \\
& v_{1}=\frac{T(0)-T(a)}{Q_{0}}=\frac{20(0)-T(t)}{Q_{0}} \\
& v_{2}=\frac{20(a)-T(a)}{Q_{0}}
\end{aligned}
$$

the expressions (3.3), when evaluated at $r=0$ and $r=a$, read

$$
\begin{aligned}
& \left|\sigma_{r}-\sigma_{\theta}\right|_{r=0}=0, \quad\left|\sigma_{r}-\sigma_{\theta}\right|_{r=a}=\left|-\frac{\alpha E^{z}}{1-\nu} v_{2} Q_{0}\right|, \\
& \left|\sigma_{r}-\sigma_{z}\right|_{r=0}=\left|\frac{\alpha E}{1-v} \frac{Q_{0} m a_{2}}{2}-R\right|, \quad\left|\sigma_{r}-\sigma_{z}\right|_{r=a}=\left|-\frac{\alpha E}{1-\nu} v_{2} Q_{0}-R\right|,
\end{aligned}
$$

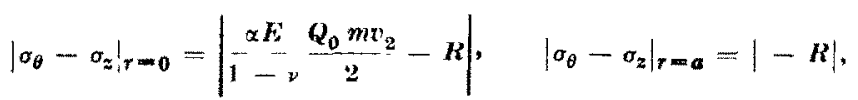

where the arbitrary constant $Q_{0}$ will be associated subsequently with a physical quantity related to the temperature field. A detailed comparison of the relative values of the five independent expressions appearing in (3.5) for the entire range of the load parameters, though straightforward, is lengthy and tedious. For our present purposes, however, we note that if the values of $m, Q_{0}$ and $F^{2}$ are restricted $t o$ the range $1 \cong m \leqq 2, Q_{0}>0$, then one is led to the result that the initiation of yield may occur in the three ways, summarized in Table 1. It may be noted here that the choice of the range of $u$ in Table 1 was motivated by the temperature distribution employed in Section 5 for which $m \simeq 1 \cdot 12$.

TABLE $1 . \quad(1 \leq m \leq 2)$

\begin{tabular}{c|c|c}
\hline Range of the parameter $R$ & Yield condition & Location of initial yield \\
\hline$R \leq \frac{\alpha E}{1-v} v_{2} Q_{0} \cdot \frac{m-2}{2}$ & $a_{r}-\sigma_{z}-2 h$ & $r=0$ \\
\hline$\frac{\alpha E}{1-v} v_{2} \cdot \frac{Q_{0}}{2}(m-2) \leqq R \leqq 0$ & $\sigma_{\theta}-\sigma_{z}-2 k$ & $r=a$ \\
\hline$R \leqq 0$. & $a_{r}-a_{\theta}=-2 k$ & $r=a$ \\
\hline
\end{tabular}




\section{Solutions in the Plastic Domain}

The determination of the state of stress in the plastic domain generally, requires the solution of the system of nonlinear differential equations (2.1) to (2.7); however, it may be shown that for a linear workhardening material these equations can be directly integrated. To this end we observe that if $f$ is a regular homogeneous function of degree $n$, then by (2.6), (2.3), and with an appeal to Euler's theorem on homogeneous functions, $W_{p}=h f n f$. When $f$ is the loading function associated with the regular regimes of $(2,4)$, then $n=1, f=2 k$, and

$$
W_{p}=2 h f k \text {. }
$$

Substitution of (4.1) into (2.5) leads to $\dot{k} \cdot k=\eta(h \dot{f})$, which upon integration becomes

$$
\log \left(k / k_{0}\right)=\eta \lambda ; \quad \lambda=\int h \dot{f} d t
$$

indicating the nonlinear variation of $k$ with $\lambda$. Expanding the left-hand side of (4.2) about $k^{\prime} k_{0}=1$ and retaining only the linear term, results in

$$
k-k_{0}(\mathbf{1}+\eta \lambda)
$$

as the law for linear hardening which, since the flow rule (2.3) was employed, is valid only for regular regimes of the Tresca yield function. The corresponding result for the singular regimes of $(\mathbf{2 . 4})$ requires different considerations, e.g., for the regime $B$ in Fig. 1, $\sigma_{\tau}=\sigma_{z^{*}} \sigma_{\theta}=\sigma_{r}+2 k,(2.6)$ because of the ranishing of plastic volume change becomes

$$
W_{p}^{r}=2 k \dot{\epsilon}_{\theta}^{\prime \prime} .
$$

Repeating the steps between (4.2) and (4.3) we obtain

$$
k=k_{0}\left(1+\eta \epsilon_{\theta}{ }^{\prime \prime}\right),
$$

which is identical in form to (4.3), except that $\lambda$ is "placed by $\epsilon_{\theta}$ ". Fxpressions similar to (4.5) may be deduced in a similar manner for other singular regimes of $(2.4)$.

The stress field is, in general, statically indeterminate, requiring the simultaneous solution of the seven equations (2.1) to (2.4), (2.7), (2.8) and one of the type (4.3) or (4.5). As this system is linear, it admits twelve solutions, corresponding to the six regular and six singular regimes of the piecewise linear loading function (2.t). These solutions for the radially symmetric stress and displacement fields are general and are valid either in full or in part for all radially symmetric temperature distributions, including the more specialized examples, where the initial yield occurs at $r=a$ or $r=0$, discussed in Section 3.

We now proceed to consider the individual regular regimes of Tresca's loading functions as follows :

(a) Regime $A B$ ( $\sigma_{z}$, the intermediate principal stress). The solution here is included in the work of BLAND (1956) and will not be repeated. It should be recalled that the workhardening parameter employed by BLAND, namely the equivalent plastic strain (see Hri. 1950, p. 30), differs from that used here (2.6); however, as shown subsequently by $B_{L A N D}(1957)$, the two parameters are equivalent 
as long as the regime in question is regular and associated with either a linear or a quadratic loading function.

(b) Regime $B C$ ( $\sigma_{r}$, the intermediate principal stress). The presence of the axial component of stress $\sigma_{z}$ in the loading function, corresponding to this regime, renders the stress distribution statically indeterminate, even in the nonhardening case. Substituting $\sigma_{z}$ from the loading function, i.e., $\sigma_{\theta}-\sigma_{z}=2 k$, and $\sigma_{\theta}$ from (2.1) into the compressibility condition (2.7), gives

$$
\frac{\partial u}{\partial r}+\frac{u}{r}+\epsilon_{z}(r, t)=\frac{1-2 \nu}{E}\left(3 \sigma_{r}+2 r \frac{\partial \sigma_{r}}{\partial r}\right)-\frac{2(1-2 \nu)}{E} k+3 \alpha T,
$$

from which

$$
\begin{aligned}
u=\frac{1-2 \nu}{E} \frac{1}{r}\left[2 r^{2} \sigma_{r}-\right. & \left.\int^{r} r \sigma_{r} d r\right]+\frac{3 \alpha}{r} \int^{r} \operatorname{Tr} d r \\
& -\frac{2(1-2 \nu)}{E} \frac{1}{r} \int^{r} k r d r-\frac{1}{r} \int^{r} \epsilon_{z} r d r+\frac{B_{1}}{r} .
\end{aligned}
$$

Throughout this section $\sigma_{\theta}$ has been assumed greater than $\sigma_{z}$; when $\sigma_{z}>\sigma_{\theta}$ the sign of $k$ need only be taken negative.

The flow law (2.3) gives upon integration with respect to time

so that

$$
\epsilon_{r}^{\prime \prime}: \epsilon_{\theta}^{\prime \prime}: \epsilon_{z}^{\prime \prime}=0: 1:-1,
$$

$$
\epsilon_{\theta}=\epsilon_{\theta}^{\prime}+\epsilon_{\theta}^{\prime \prime}=\epsilon_{\theta}^{\prime}-\epsilon_{z}^{\prime \prime}=\epsilon_{\theta}^{\prime}-\epsilon_{z}+\epsilon_{z}^{\prime} .
$$

By the generalized Hooke's law together with (2.1) and the loading function, the total tangential strain given above may be expressed as

$$
\epsilon_{\theta}=\frac{u}{r}=-\epsilon_{z}+\frac{2(1-\nu)}{E} \cdot\left(\sigma_{r}+r \frac{\partial \sigma_{r}}{\partial r}-k\right)-\frac{2 \nu}{E} \sigma_{r}+2 \alpha T .
$$

Elimination of $u$ betwecn (4.6) and (4.7) after some manipulation results in the following differential equation for $\sigma_{r}$ :

$$
\begin{aligned}
& 2(1-\nu) r^{2} \frac{\partial^{2} \sigma_{r}}{\partial r^{2}}+6(1-\nu) r \frac{\partial \sigma_{r}}{\partial r}+(1-2 \nu) \sigma_{r}= \\
& -\alpha E T-2 \alpha E r \frac{\partial T}{\partial r}+2 k+2(1-\nu) r \frac{\partial k}{\partial r}+E \epsilon_{z}+r E \frac{\partial \epsilon_{z}}{\partial r} .
\end{aligned}
$$

It may be of interest to note that (4.8) may also be obtained from the appropriate differential equation of strain compatibility.

Since the yield stress $k$ in general depends on parameters which may in turn be functions of the dependent variable $\sigma_{r},(4.8)$ cannot be solved until $k$ is specified. The nature of the loading, however, is such that the axial strain is independent of $\sigma_{r}$, so that the solution of (4.8) is immediately attainable for a nonhardening material. The homogeneous solution of (4.8) for a nonhardening material is of the form

$$
\left.\left(\sigma_{r}\right)_{h}=\frac{A_{1}}{r^{s_{1}}}+\frac{A_{2}}{r^{s_{2}}}, \quad \text { where } \quad s_{1}\right]=1 \mp \frac{1}{\sqrt{2(1-v)]}},
$$

and the particular solution may be found by variation of parameters to be 
The elastic-plastic eylinder subjected to radially distributed heat source

$$
\begin{aligned}
& \frac{\left(\sigma_{r}\right)_{p}}{k}=\frac{2}{1-2 \nu} \\
& +\underset{2(1-\nu)\left(s_{2}-s_{1}\right)}{2}\left\{\frac{1+2 s_{2}}{r^{s_{2}}} \int \frac{T}{T_{a}} r^{s_{2}-1} d r-\frac{1+2 s_{1}}{r^{s_{1}}} \int \frac{T}{T_{a}} r^{s_{1}-1} d r\right\} \\
& +\frac{E}{2(1-\nu)\left(s_{2}-s_{1}\right)}\left\{\frac{1}{r^{s_{1}}} \int \frac{\partial\left(r \epsilon_{z}\right)}{\partial r} r^{s_{1}-1} d r-\frac{1}{r^{s_{2}}} \int \frac{\partial\left(r \epsilon_{z}\right)}{\partial r} r^{s_{2}-1} d r\right\}
\end{aligned}
$$

where

$$
\Phi=\frac{\alpha E T_{\mathrm{a}}}{k_{0}}
$$

With $\sigma_{r}$ known the tangential and the axial stress may be found through the equilibrium equation and the yield condition, respectively. The second expression in braces $(4.10)$ leads to a term appearing in the expression for $\sigma_{z}$ which may be identified with $C_{3}$, and results in

$$
\epsilon_{2}=2(1-\nu) \frac{k_{0}}{E} s_{1} s_{2} C_{3}+\frac{C_{4}}{r}
$$

where $C_{4}$ is a function of time only. Thus, the stress distribution for a nonhardening material is given by

$$
\begin{aligned}
\frac{\sigma r}{k_{0}} & =\frac{A_{1}}{r^{s_{1}}}+\frac{A_{2}}{r^{s_{2}}}+C_{3}+\frac{2}{1-2 \nu} \\
& -\frac{\Phi}{2(1-\nu)\left(s_{1}-s_{2}\right)}\left[\frac{1+2 s_{2}}{r^{s_{2}}} \int \frac{T}{T_{a}} r^{s_{2}-1} d r-\frac{1+2 s_{1}}{r^{s_{1}}} \int \frac{T}{T_{a}} r^{s_{1}-1} d r\right] \\
\frac{\sigma_{\theta}}{k_{0}} & =\left(1-s_{1}\right) \frac{A_{1}}{r^{s_{1}}}+\left(1-s_{2}\right) \frac{A_{2}}{r^{s_{2}}}+C_{3}+\frac{2}{1-2 v} \\
& -\frac{\Phi}{2(1-\nu)\left(s_{1}-s_{2}\right)}\left[\frac{\left(1-s_{2}\right)\left(1+2 s_{2}\right)}{r^{s_{2}}} \int \frac{T}{T_{a}} r^{s_{2}-1} d r\right. \\
& -\frac{\left(1-s_{1}\right)\left(1+2 s_{1}\right)}{r^{s_{1}}}\left[\frac{T}{T_{a}} r^{s_{1}-1} d r+2\left(s_{2}-s_{1}\right) \frac{T}{T_{a}}\right] \\
& \frac{\sigma_{z}}{k_{0}}=\frac{\sigma_{\theta}}{k_{0}}-2 .
\end{aligned}
$$

Since the foregoing solution is not valid when $\nu=\frac{1}{2}$, the integrations leading to (4.12) requires separate consideration. Here the solution for an incompressible material may be obtained directly from $(4.8)$; the result is similar to $(4.12)$, the chief difference being that the leading terms are logarithmic in $r$, and that with reference to $\epsilon_{z}$, corresponding for (4.11), a uniform axial stress gives rise to a uniform axial strain. These results, for an uncompressible material, when differentiated with respect to time, agree with the previously known solution (WEINER 1959) for the stress-rate and the displacement-rate of an incompressible material. In contrast to the solution for an incompressible material, it may be seen from (4.11) that for a compressible material a uniform axial stress may result in a nonuniform axial strain. 
(c) Regime $C D\left(\sigma_{\theta}\right.$, the intermediate principal stress). As in (b) the stress distribution is again statically indeterminate, and the procedure leading to (4.6) and (4.8) gives

$$
\begin{aligned}
& u=\frac{1-2 \nu}{E^{\prime}} \cdot \int^{r}\left(3 r \sigma_{r}+r^{2} \frac{\partial \sigma_{r}}{\partial r}\right) d r-\frac{2(1-2 \nu)}{E r} \int^{r} k r d r \\
& \left.+\frac{3 \alpha}{r} \int^{r} \operatorname{Tr} d r-\frac{E}{r} \int^{r} r \epsilon_{z} d r+\frac{B_{2}}{r},\right\} \\
& r^{2} \frac{\partial^{2} \sigma_{r}}{\partial r^{2}}+3 r \frac{\partial \sigma_{r}}{\partial r}-(1-2 v) \sigma_{r}=-2 k-2 \nu r \frac{\partial k}{\partial r} \\
& \left.-E \epsilon_{z}+\alpha E T-\alpha E \frac{\partial T}{\partial r} r,\right\}
\end{aligned}
$$

whose solution for a nonhardening material is

$$
\begin{aligned}
& \frac{\sigma_{r}}{k_{0}}=\frac{A_{3}}{r^{r_{3}}}+\frac{A_{4}}{r^{s_{4}}}+C_{3}-\frac{2}{1-2 v} \\
& +\frac{\Phi}{s_{3}-s_{4}}\left[\frac{1+s_{4}}{r^{s_{4}}} \int \frac{T}{T_{a}} r^{s_{4}-1} d r-\frac{1+s_{3}}{r^{s_{3}}} \int \frac{T}{T_{a}} r^{s_{3}-1} d r\right] \\
& \frac{\sigma_{\theta}}{k_{0}}=\left(1-s_{3}\right) \frac{A_{3}}{\gamma_{3}}+\left(1-s_{4}\right) \frac{A_{4}}{r^{s_{4}}}+C_{3}-\frac{2}{1-2 \nu} \\
& +\underset{s_{3}}{\frac{\Phi}{-s_{4}}}\left[1-s_{3}^{2} \int \frac{T}{r^{s_{3}}} r^{s_{3}-1} d r-\frac{1-s_{4}^{2}}{r^{s_{4}}} \int \frac{T}{T_{a}} r^{s_{4}-1} d r+\left(s_{4}-s_{3}\right) \frac{T}{T_{a}},\right. \\
& \frac{\sigma_{z}}{k_{0}}=\frac{\sigma_{r}}{k_{0}}-2 \text {. }
\end{aligned}
$$

The states of stress for the singular regimes $A, B, C, D, E$ and $F$ of Fig. 1 are also, in general, statically indeterminate for a workhardening material; for a nonhardening material, however, this is not the case, and the solutions may be obtained directly from the equilibrium equation and the yield condition:

$$
\begin{aligned}
& \text { regime } A:\left\{\begin{array}{l}
\sigma_{r}=2 k_{0} \log r+D_{1} \\
\sigma_{\theta}-\sigma_{z}=\sigma_{r}-2 k_{0}
\end{array}\right. \\
& \text { regime } B:\left\{\begin{array}{l}
\sigma_{r}=\sigma_{z}=2 k_{0} \log r+D_{2} \\
\sigma_{\theta}=\sigma_{z}-2 k_{0}
\end{array}\right. \\
& \text { regime } C:\left\{\begin{array}{l}
\sigma_{r}=\sigma_{\theta}=D_{3} \\
\sigma_{r}=D_{3}-2 k_{0}
\end{array}\right.
\end{aligned}
$$

where $D_{1}, D_{2}, D_{3}$ are functions of time only. The states of stress at regimes $D, E$ and $F$ are obtained by replacing $k_{0}$ by $-k_{0}$ in (4.16), (4.17) and (4.18), respectively. The radial displacement associated with the above solutions, again obtained from (2.7), reads

$$
u=\frac{\zeta_{1} r}{2}+\zeta_{2} k_{0} \frac{r}{2}+\frac{6 \zeta_{3} k_{0}}{r}(r \log r-r)+\frac{3 \alpha}{r} \int^{r} T r d r+\frac{B_{3}}{r}
$$


where $B_{3}$ is an arbitrary function of time and the coefficients $\zeta_{1}, \zeta_{2}$ and $\zeta_{3}$ for each of the singular regimes may be found from

$$
\frac{1-2 \nu}{E}\left(\sigma_{r}+\sigma_{\theta}+\sigma_{z}\right)=\zeta_{1}+\zeta_{2} k_{0}+6 \zeta_{3} k_{0} \log r
$$

together with (4.16), (4.17) and (4.18).

\section{PART II. APPLICATION TO CYLINDRICAL NUCLEAR REACTOR FUEL ELEMENTS}

\section{Temperature Distribution and the Cifaracter of Initial Yifld}

The fuel portion of a cylindrical reactor fuel element can be treated as a solid cylinder in the state of plane strain subjected to a thermal gradient and an external pressure ; the former is due to heat generated by the nuclear fission of the element itself which thus behaves as a distributed heat source, while the latter is due to the expansion of the solid fissionable core against the nonfissionable hollow cylindrical claclding, which serves to conduct heat away. The pressure $p$ at the interface $(r=a)$ of the two cylinders is, therefore, not independent but rather a consequence of the temperature field. It is assumed for clarity that when $Q$ is zero the stress field vanishes, i.e., there is no shrink-fit, although this may be easily included. A complete analysis of the reactor problem entails an elastic-plastic treatment of both cylinders, but here emphasis is placed on the elastic-plastic solid core and the parameters of the problem are selected with this goal in mind; the hollow cladding is mentioned only to the extent that it affects the solution of the solid core.

The heat source will be taken as Gaussian in character, i.e.,

$$
Q=Q_{0} \exp \left(-\mu r^{2}\right)
$$

where $\mu^{2}$ is a parameter. When $\mu^{2}=0, Q$ reduces to $\boldsymbol{Q}_{0}$, a distributed source of uniform strength. With (5.1) the solution of (2.8), subject to the boundary condition $T=T a$ at $r=a$ and the regularity requirement at $r=0$, may he written as

where

$$
T=T_{a}+\frac{Q_{0}}{4 K_{1} \mu^{2}}\left[E i\left(-\mu^{2} r^{2}\right)-E i\left(-\mu^{2} a^{2}\right)-2 \log \frac{r}{a}\right],
$$

$$
\boldsymbol{E} i(-x)=-\int_{x}^{\infty} \frac{e^{-y}}{y} d y
$$

is the exponential integral.

Since there is no heat source in the cladding $(a \leqq r \leqq b)$, the temperature distribution is given by the homogeneous solution of (2.8), i.e.,

$$
T=\frac{T_{b}-T_{a}}{(\log b / a)} \log \frac{r}{a}+T_{a}
$$

where the boundary conditions $T_{a}$ and $T_{b}$ have been utilized.

Before determining the location of the initial yield, as well as $p^{*}, T^{*}$ and $Q_{0}{ }^{*}$ (i.e., the values. respectively, of $p, T$ and $Q_{0}$ at the initiation of yield), it is necessary 
to establish the functional relation between the parameters $Q_{0}, T_{a}, F$ and $p$. One such relation is obtained by equating the radial displacements of the two cylinders at their interface $r=a$. Since prior to yielding both cylinders are entirely elastic. the displacements in each may be written from the first two of (3.1) as

$$
u^{(i)}=-\frac{1+\nu_{i}}{E i} r \sigma_{r}^{(i)}+2 C_{1}^{(i)} r \frac{1-v_{i}}{1-2 \nu_{i}}
$$

where the index $i=1,2$, refers to the quantities appropriate to the solid core and the hollow cladding, respectively. If the pressure at the inner face is $-p$, then by (5.5)

$$
p\left[\frac{1+\nu_{1}}{E_{1}}-\frac{1+\nu_{2}}{E_{2}}\right]=\frac{2\left(1-\nu_{2}\right)}{1-2 \nu_{2}} C_{1}^{(2)}-2\left(1-\nu_{1}\right) C_{1}^{(1)}
$$

and the coefficients $C_{1}{ }^{(t)}$ are found from the second of $(3.1)$ and the boundary conditions $\sigma_{r}(a)=-p, \sigma_{r}(b)=0$ :

$$
\begin{aligned}
& C_{1}^{(1)}=\left(1-\frac{\left.2 v_{1}\right)\left(1+v_{1}\right)}{F_{1}}\left[-p+\frac{\alpha_{1} E_{1}}{1} \theta(a)\right]\right. \\
& C_{1}{ }^{(2)}=\frac{\left(1-2 \nu_{2}\right)\left(1+v_{2}\right)}{E_{2}}\left[-p\left(1-\frac{1}{1-a^{2} b^{2}}\right)+\frac{a_{2} E_{2} T_{a}}{1-v_{2}}\left(\frac{\theta(b)}{T_{a}}\right) i-\frac{1}{a^{2} b^{2}}\right]
\end{aligned}
$$

where by $(5.2)$ and $(5.4)$

$$
\theta(a)=\frac{1}{a^{2}} \int_{0}^{a} \operatorname{Tr} d r=\frac{T_{a}}{2}+\frac{Q_{0}}{8 K_{1} \mu^{2}}\left(1+\frac{\exp \left(-\mu^{2} a^{2}\right)-1}{\mu^{2} a^{2}}\right)
$$

and similarly,

$$
\theta(b)=\frac{1}{b^{2}} \int_{a}^{b} \operatorname{Tr} d r=\frac{T_{a}}{2}\left\{\frac{T_{b}}{T_{a}}+\left(\frac{T_{b}}{T_{a}}-1\right)\left[\frac{a^{2} / b^{2}-1+2 \log b / a}{2 \log b / a}\right]\right\}
$$

The relation between $Q_{0}$ and the interface temperature $T_{a}$, following the procedure employed by Grasstone (1955) for the case $\mu^{2}=0$, is obtained by equating the flux per unit length, produced in the core at $r=a$,

$$
q(a)=-\frac{\pi Q_{0}}{\mu^{2}}\left[\exp \left(-\mu^{2} a^{2}\right)-1\right]
$$

to that across any surface of the source-free cladding

Hence

$$
q=\frac{\left(T_{a}-T_{b}\right)}{\log b / a} 2 \pi K_{2}
$$

where

$$
T_{a}=h Q_{0}
$$

$$
h=\frac{\log (b / a)}{\left(T_{b} / T_{a}-1\right) 2 \mu^{2} K_{2}} \cdot\left[\exp \left(-\mu^{2} a^{2}\right)-1\right]
$$

which reduces to GLAsstone's results when $\mu^{2}=0$. 
The elastic-plastic eylinder subjected to radially distributed heat source

Substituting for $C_{1}{ }^{(1)}$ and $C_{1}{ }^{(2)}$ from (5.7), equation (5.6) becomes

$$
\begin{aligned}
& p\left[\frac{1+v_{1}}{E_{1}}-\frac{1+\nu_{2}}{E_{2}}-\frac{2\left(1-\nu_{2}^{2}\right)}{E_{2}\left(b^{2} / a^{2}-1\right)}-\frac{2\left(1-\nu_{1}{ }^{2}\right)}{E_{1}}\right] \\
& =Q_{0}\left[2\left(1+\nu_{2}\right) \alpha_{2} h\left(\begin{array}{c}
\theta(b) \\
T_{a}
\end{array}\right) \cdot 1-a^{2} b^{2}-\alpha_{1}\left(1+\nu_{1}\right)\left(h+\nu_{2}\right)\right],
\end{aligned}
$$

where $h$ and $y_{2}$ are defined by $(5.11 \mathrm{~b})$ and $(3.4 \mathrm{~b})$, respectively.

We are now in a position to establish the character of initial yield. Since in the state of plane strain the superposed axial stress $C_{3}=0$, we observe by the last of (3.1) that when $\rho=0$,

$$
\frac{F}{\pi a^{2}}=-2 \alpha_{1} E_{1} \theta(a)-2 \nu_{1} p
$$

It then follows from Table 1 and the first of (3.4a) that initial yielding occurs at $r=0$ if

$$
-\alpha_{1} E_{1} T_{a}-\alpha_{1} E_{1} \nu_{2} Q_{0}+p\left(1-2 \nu_{1}\right)<\frac{\alpha_{1} E_{1}}{1-\nu_{1}} \nu_{2} Q_{0} \frac{m-2}{2}
$$

in which case (as may be seen from Table 1) the yield condition is characterized by

$$
\left|\sigma_{\theta}-\sigma_{z}\right|=\left|\sigma_{r}-\sigma_{z}\right|=2 k_{0} .
$$

Before proceeding further it is necessary to specify the ratios of the coefficients (both mechanical and thermal) in the core to those in the cladding, i.e.,

$$
\frac{E_{1}}{E_{2}}=2, \quad \frac{\alpha_{1}}{\alpha_{2}}=2, \quad \frac{k_{2}}{k_{1}}=8, \quad \frac{\nu_{1}}{\nu_{2}}=1, \quad=0.22,
$$

as well as

$$
\frac{T_{a}}{T_{b}}=\frac{3}{2}, \quad \frac{b}{a}=\frac{3}{2}
$$

It may be noted here that the selection of the ratios $(5.16 a)$ is based on the assumption of a uranium core surrounded by an aluminium cladding.

If, with the aid of (5.12) and (5.11), $p$ and $T_{a}$ are eliminated from (5.14), then it may be easily shown that the ratios (5.16) render the inequality (5.14) valid. Hence if yielding occurs in the core before the cladding-which, subject to a later verification. is the case for the selected ratios (5.16) - then it must occur at $r=0$ according to (5.15).

Substituting $F$ from (5.13) into the second of (3.5) and equating the results to $2 k_{0}$ in accord with $(5.15)$ results in the following relation between $Q_{0}{ }^{*}$ and $p^{*}$,

$$
\frac{\alpha_{1} E_{1}}{2\left(1-\nu_{1}\right)} Q_{0}^{*}\left[2 h\left(1-\nu_{1}\right)+\nu_{1}+\nu_{2}\left(1-2 \nu_{1}\right)\right]-p^{*}\left(1-2 v_{1}\right)=2 k_{0}
$$

which may be solved together with (5.12) to obtain independent expressions for $p^{*}$ and $Q_{0}^{*}$.

To confirm the statement that the solid core yields before the hollow cladding we need only compare $p^{*}$ with the corresponding value of $p$ obtained under the assumption that initial yield first occurs in the cladding instead. This value $p^{*}$ is found by incorporating (5.12) into the results of BLAND (1956), where it is sub- 
sequently found that $p^{*}<\bar{p}^{*}$ for the parameters selected, so that yielding occurs first in the core. It is important to observe that having established the character of initial yield, the value of $\bar{p}^{*}$ ceases to be valid even for subsequent yielding of the cladding, since it was obtained through the use of the relation (5.12) which holds only when both elements are completely elastic. An exact value of $\bar{p}^{*}$ may be obtained using a relation similar to $(\mathbf{5 . 1 2})$ but reflecting the true state of plastic deformation in the core.

\section{Eiantic-Plastic Solution for linear Workilardening Material.}

Having determined the character of the initial yield, we now proceed to establish the mode of the plastic deformation in the solid core. Because of the symmetry the elastic-plastic boundary is a cylinder of radius $\rho$, defining the plastic $(0 \leqq r \leqq \rho)$ and the elastic ( $\rho \fallingdotseq r \supseteq a)$ domains. The solution in the elastic domain offers no difficulty and may be obtained from (3.1) by setting $C_{3}=0$. For the plastic domain all the possible fields of stress discussed in Section 4 are at our disposal. Attempts to characterize the state of stress in the plastic domain by a single regime (although satisfying all of the field equations) disclose that such solutions do not conform to all of the continuity requirements concerning the stresses and displacements at the elastic-plastic boundary and hence must be rejected. These continuity requirements, together with the consideration of (a) the loading paths during the purely elustic stage of deformation (Fig. 2) for any $r<0$,

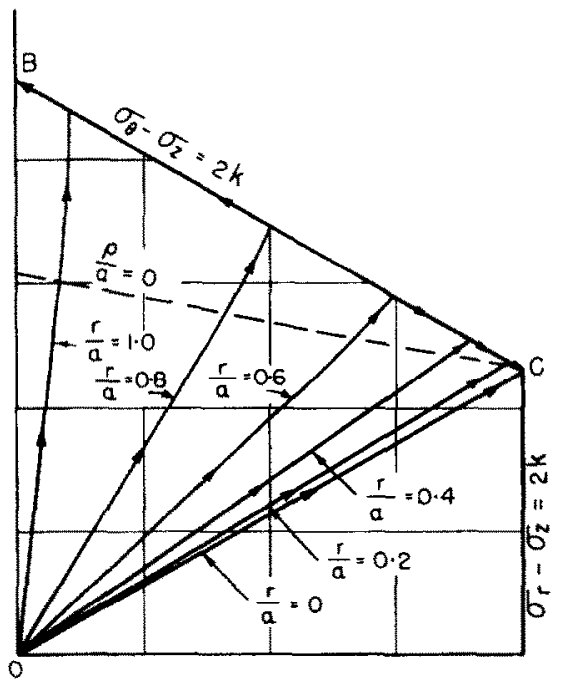

Fig. 2. A portion of Tresca's yield surface showing the stress profles for a nonhardening material $(\gamma=0)$. The dashed curve corresponds to the state of stress at the initiation of yield.

(b) the fact that the initial yield occurs on an edge of the Tresca yield surface (the singular regime $C$ ), and (c) the possible paths the state of stress may take following the initial yield, lead to the possibility of regarding the plastic domain $(0 \leqq r \leqq \rho)$, as consisting of two concentric zones, separated by an interface 
The elastic-plastic cylinder subjected to radially distributed heat source

$r=\rho_{1}$, and with the state of stress in each zone characterized by a different regime of Tresca's loading function. For reasons that will become apparent later, the solution in the range of contained plastic deformation of the cylinder will be subsequently referred to as the solution for Stage I of deformation.

Stage $I$

The central zone $I\left(0 \leqq r \leqq \rho_{1}\right)$ consists of stress states lying along an edge (regime $C$ in Fig. 1) and are given by equations (4.18). The state of stress in the plastic annulus, zone II ( $\left.\rho_{1} \leqq r \leqq \rho\right)$, surrounding zone I, lies on the face

$$
\sigma_{\theta}-\sigma_{z}=2 k
$$

of the Tresca surface and thus satisfies equation (4.8) whose solution for a nonhardening elasticplastic material is given by (4.12). However, before the stress field in zone II may be found for a workhardening material, the yield stress $k$ appearing in $(4.8)$ must be determined through the workhardening law (4.3), which by the flow rule (2.3) and for the state of plane strain $\left(\epsilon_{z}=0\right)$ becomes

$$
k=k_{0}\left(1+\eta \epsilon_{\mathrm{z}}{ }^{\prime}\right) .
$$

With the use of the generalized Hooke's law, $(2.1)$ and $(6.1)$, the elastic component of the axial strain may be written as

$$
\epsilon_{z}^{\prime}=\frac{1}{E_{1}}\left\{\left(1-v_{1}\right) r \frac{\partial \sigma_{r}}{\partial r}+\left(1-2 v_{1}\right) \sigma_{r}-2 k-\alpha_{1} E_{1}^{\prime} T^{\prime}\right\}
$$

so that by (6.2) $k$ assumies the form

$$
\frac{k}{k_{0}}=\frac{1}{1+\gamma}+\frac{\kappa}{2}\left[\left(1-\nu_{1}\right) r \frac{\partial}{\partial r}\left(\frac{\sigma_{r}}{k_{0}}\right)+\left(1-2 \nu_{1}\right) \frac{\partial_{r}}{k_{0}}+\Phi_{1} \frac{T}{T_{a}}\right],
$$

where

and

$$
\gamma=\frac{2 \eta k_{0}}{\boldsymbol{E}_{\mathbf{1}}}, \quad \kappa=\frac{\gamma}{1+\gamma}
$$

$$
\Phi_{1}=\frac{\alpha_{1} E_{1}^{\prime} T_{a}}{k_{0}}
$$

When (6.4) is substituted into (4.8), there results the following differential equation in $\sigma_{r}$ :

$$
\begin{aligned}
\left.b_{1} r^{2} \frac{\partial^{2}}{\partial r^{2}}\left(\frac{\sigma_{r}}{k_{0}}\right)+3 b_{1} r \frac{\partial}{\partial r}\left(\frac{\sigma_{r}}{k_{0}}\right)+b_{2} \frac{\sigma_{r}}{k_{0}}=\frac{2}{1+\gamma}-\Phi_{1} \frac{T}{T_{a}} \frac{1}{1+\gamma}\right] \\
+\Phi_{1} r \frac{\partial}{\partial r}\left(\frac{T}{T_{a}}\right)\left[\left(1-v_{1}\right) \kappa-2\right],
\end{aligned}
$$

where

$$
\begin{aligned}
& b_{1}=2\left(1-v_{1}\right)-\left(1-v_{1}\right)^{2} \kappa, \\
& b_{2}=\left(1-2 v_{1}\right)(1-\kappa) .
\end{aligned}
$$

The homogeneous solution of (6.6) has the same form as (4.8) with $s_{1}$ and $s_{2}$ in (4.9) replaced by

$$
\left.\left.\begin{array}{l}
s_{5} \\
s_{6}
\end{array}\right\}=1 \mp \sqrt{\left(\frac{b_{1}}{b_{1}}-b_{2}\right.}\right),
$$

respectively; these reduce to $s_{1}$ and $s_{2}$ when $\eta=0$.

The particular solution of (6.6) for any temperature distribution, as in Section 4, may be obtained by variation of parameters. However, since the character of the initial yield was established only for the class of temperature distributions for which $1 \leqslant m \leqslant 2$ (Table 1), in the 
complete solution of $(6.6)$ recorded below the specife temperature distribution (5,2) corresponding $m \simeq 1 \cdot 12$ has been used:

$$
\begin{aligned}
& \frac{\sigma_{r}}{k_{0}}=\frac{A_{5}}{r^{s_{5}}}+\frac{A_{6}}{r^{s_{6}}}+\frac{2-\Phi_{1}}{1-2 \nu_{1}} \\
& -\frac{\Phi_{1}}{2 K_{1} \mu^{2} h} \int \frac{s_{5}+s_{6}}{s_{5} s_{6}}(1+\gamma)\left[\left(1-v_{1}\right) \kappa-2\right] \\
& +E i\left(-\mu^{2} r^{2}\right)-E i\left(-\mu^{2} a^{2}\right)-2 \log \frac{r}{a} \\
& -\frac{1}{2(1-2 v)\left(s_{5}-s_{6}\right)}\left[\frac{s_{5}\left[1-2 s_{6}+\left(1-v_{1}\right) s_{6} k\right]}{\mu^{s} r_{6}^{s_{6}}} \Gamma_{x}\left(s_{5} / 2\right)\right. \\
& \left.-s_{6}\left[1-\frac{\left.2 s_{5}+\left(1-\nu_{1}\right) s_{5} \kappa\right]}{\mu^{s} r^{s}} \Gamma_{x}\left(s_{5} / 2\right)\right]\right\} \\
& \frac{\sigma_{\theta}}{k_{0}}=\left(1-s_{5}\right) \frac{t_{5}}{r_{5}^{s_{5}}}+\left(1-s_{6}\right) \frac{A_{6}}{r_{6}}+\frac{2-\Phi_{1}}{1-2 v_{1}} \\
& -\frac{\Phi_{1}}{2 K_{1} \mu^{2} h}\left\{\frac{s_{5}+s_{6}}{s_{5} s_{6}}-\left(1-(1+\gamma)\left[\left(1-\nu_{1}\right) \kappa-2\right]\right)\right. \\
& +k i\left(-\mu^{2} r^{2}\right)-k i\left(-\mu^{2} a^{2}\right)-2 \log \frac{r}{a} \\
& -\frac{1}{2\left(1-\frac{1}{\left.2 v_{1}\right)\left(s_{5}-s_{6}\right)}\left[s_{5}\left(1-s_{6}\right)\left[1-\frac{2 s_{6}}{\mu_{6} s_{6}}+\left(1-v_{1}\right) s_{6} \kappa\right]\right.\right.} \Gamma_{x}\left(s_{6} / 2\right) \\
& \left.-\frac{s_{6}\left(1-s_{5}\right)\left[1-2 s_{5}+\left(1-v_{1}\right) s_{5} \kappa\right]}{\mu^{s_{5}} r^{p_{5}}} \Gamma_{x}\left(s_{5} / 2\right)\right] \mid \\
& \frac{\sigma_{\tilde{*}}}{k_{0}}=\frac{\sigma_{\theta}}{k_{0}}-\frac{2 k}{k_{0}}
\end{aligned}
$$

where

$$
\Gamma_{x}(n)=\int_{0}^{x=\mu^{2} r^{2}} c^{-y} y^{n-1} d y
$$

is the incomplete gamma function. It follows from (6.1) and (2.3) that for loading from an elastic state $\dot{\epsilon}_{r}^{\prime \prime}=0$, and hence the radial strain is purely elastic. Thus, the radial displacement corresponding to the solution $(6.8)$ is most easily evaluated through $(2.7)$ with $\epsilon_{z}=0$, the generalized Hooke's law, and (6.1) by

$$
\frac{\boldsymbol{E}_{1} u}{r}-2 v_{1} \sigma_{r}+2\left(1-v_{1}\right) \sigma_{\theta}-2\left(1-v_{1}\right) k+2 \alpha_{1} E_{1} T
$$

which with (6.8) completes the solution for zone $\amalg\left(p_{1}=r \fallingdotseq p\right)$

Summarizing, the temperature field is given throughout the region $0 \doteq r \equiv a$ by $(5,2)$, the stress and displacement fields are given:

(a) in plastic zone $I\left(0 \cong r \cong \rho_{1}\right)$ by $(4.18)$ and (1.19), respectively, and represented in stress space by the singular regime $C$ in Fig. 1 ; (b) in plastic zone II ( $p_{1} \leqq r \leqq \rho$ ) by $(6.8)$ and (6.9) respectively, and represented in stress space by the regime $B C$; and (c) in the elastic zone $(\rho \leq r \leq a)$ by (3.1) with $C_{3}=0$.

With the form of the stress and displacement fields established in all three zones of the problem, it remains to determine the five coefficients $D_{3}, A_{2}, A_{1}, C_{1}, C_{2}$, as well as the relation between the boundaries $\rho, \rho_{1}$, and the load parameters $p$ and $\Phi_{1}$. Equilibrium together with continuity 
The elastic-plastic cylinder subjected to radially distributed heat soure

of the yield condition and displacements requires that the stresses themselves be continuous not only at the elastic-plastic boundary $\rho$ but at the interface $\rho_{1}$ between the two plastic zones. Furthermore, an examination of the compressibility equation reveals that continuity of $u$ at $r=\rho_{1}$ is equivalent to the requirement that $\epsilon_{r}^{\prime \prime}\left(\rho_{1}\right)=0$ : this latter condition ronsilembly simplifies the determination of the five coefficients mentioned above. In order to determine the foregoing unknowns, we require (a) continuity only of $\sigma_{r}, \sigma_{\theta}$, and $\epsilon_{r} "$ at $r=\rho_{1}$, since $\sigma_{z}$ and $n$ will automatically be continuous through the yield condition, and the compressibility equation. respectively; (b) continuity of $\sigma_{r}, \sigma_{\theta}$, and $\sigma_{z}$ at $r=\rho$, since $n$ is continuous again through the compressibility equation and the vanishing of $\epsilon_{r}^{\prime \prime}$ in zone II ; (c) satisfaction of the boundary condition $\sigma_{r}(a)=-p$ : and (d) the matching of the displacements of the solid cylinder with that of the hollow cladding, as was done previously in Section 5 for the purely elastic solution of the problem. For a given value of $\rho$ this stage of the problem is thus statically indeterninate, requiring the solution of eight equations in eight unknowns.

In the central zone I the stresses through equilibrium and the loating function are found to have the same form as (4.18) except that $k_{0}$ is replaced by $k$; the radial displacement is found through the compressibility equation (2.7) to be

$$
t=\frac{3}{2} \frac{1-2 v_{1}}{E_{1}} r-\frac{2\left(1-2 v_{1}\right)}{E_{1}} \frac{1}{r} \int_{0}^{r} k r d r . \quad+\frac{1}{r} \int_{0}^{r} 3 \alpha \operatorname{Tr} d r
$$

from which with the use of Hooke's law and the plastic component of radial strain is found to be

$$
\epsilon_{r}^{\prime \prime}=\frac{1-2 \nu_{1}}{2 E_{1}} D_{3}-\frac{2 h}{E_{1}}\left(1-\nu_{1}\right)+\frac{\left(1-2 \nu_{1}\right)}{E_{1}} \frac{2}{r^{2}} \int_{0}^{r} k r d r+2 \alpha_{1} T-\frac{3 \alpha_{1}}{r^{2}} \int_{0}^{r} T r d r
$$

It may be seen from the enmpressibility relation (2.7) which holds throughout the cylinder, that since the stresses and displacement $u$ are continuous at $r=\rho_{1}$, the elastic as well as the plastir components of the radial strain eich must be continuous separately. Since $\epsilon_{r}{ }^{\prime \prime}=0$ throumhunt zone II. then by (6.11)

$$
\frac{1-2 v_{1}}{E_{1}} \frac{D_{3}}{2}=\frac{2 k\left(\rho_{1}\right)}{E_{1}}\left(1-v_{1}\right)-\frac{\left(1-2 v_{1}\right)}{E_{1}} \frac{2}{\rho_{1}^{2}} \int_{0}^{\rho_{1}} k r d r-2 \alpha_{1} T\left(\rho_{1}\right)+\frac{3 \alpha_{1}}{\rho_{1}^{2}} \int_{0}^{\rho_{1}} T r i r
$$

Substituting $(4.18)$ into $(6,2)$, though Hooke's law, the value of $k$ throughout zone 1 is foumd to be

$$
\frac{k}{k_{0}}(1+\gamma)=1+\frac{\gamma}{2}\left(1-2 v_{1}\right) \frac{D_{3}}{k_{0}}+\frac{\Phi_{1} \gamma}{2} \frac{T(r)}{T_{a}}
$$

which, when solved with (6.12) after some simplification, yields

$$
\begin{aligned}
& \frac{1-2 \nu_{1}}{2} \frac{D_{3}}{k_{0}}=1-2 \Phi_{1} \frac{T\left(\rho_{1}\right)}{T_{a}}+3 \Phi_{1} \frac{\theta\left(\rho_{1}\right)}{T_{a}}+\gamma\left(1+v_{1}\right) \Phi_{1}\left[2 \frac{\theta\left(\rho_{1}\right)}{T_{a}}-\frac{T\left(\rho_{1}\right)}{T_{a}}\right], \\
& \frac{k}{k_{0}}(1+\gamma)=1+\gamma\left[1-2 \Phi_{1} \frac{T\left(\rho_{1}\right)}{T_{a}}+\frac{\Phi_{1}}{2} \frac{T(r)}{T_{a}}+\frac{3 \Phi_{1}\left(\rho_{1}\right)}{T_{a}}\right]+ \\
& +\gamma^{2}\left[2 \frac{\theta\left(\rho_{1}\right)}{T_{a}}-\frac{T\left(\rho_{1}\right)}{T_{a}}\right]\left(1+v_{1}\right) \Phi_{1}
\end{aligned}
$$

With $D_{3}$ and $k$ known, the radial displacement may now be written as

$$
\frac{E_{1} u}{r k_{0}}=\frac{1-2 \nu_{1}}{2} \frac{D_{3}}{k_{0}}\left[3-\left(1-2 \nu_{1}\right) \kappa\right]-\frac{1-2 v_{1}}{1+\gamma}+\Phi_{1} \frac{\theta(r)}{T_{\alpha}}\left[3-\left(1-2 \nu_{1}\right) \kappa\right],
$$

which completes the solution for zone I.

Continuity of the plane components of stress [given by (3.1) in the elastic zone, and by (6.8) in the plastic zone II, and by (4.18) in the plastic zone I] at $r=\rho_{1}$ and $r=\rho$ gives, respectively. 


$$
\begin{aligned}
& f_{5}=\frac{\rho_{5}}{s_{5}-s_{6}}\left[\gamma_{1}\left(1-s_{5}\right)-\beta_{1}\right], \\
& ._{6} \frac{\rho_{6}}{s_{5}-s_{6}}\left[\gamma_{1}\left(1-s_{6}\right)+\beta_{1}\right] .
\end{aligned}
$$

where

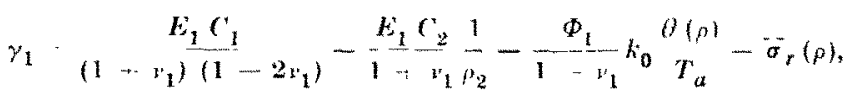

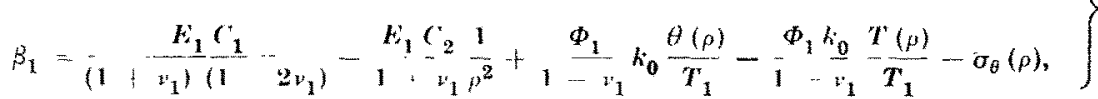

and

where

$$
f_{5}-\frac{p_{1}{ }^{6}}{s_{5}-s_{6}}\left[\gamma_{2}\left(1-s_{6}\right)-\beta_{2}\right], \quad f_{6}=\frac{\rho_{1} \phi_{6}}{s_{5}-s_{6}}\left[\gamma_{2}\left(1-s_{6}\right)+\beta_{2}\right]
$$

$$
\gamma_{2}=D_{3} \quad \sigma_{r}\left(\rho_{1}\right) \quad \beta_{2} \quad l_{3}-\sigma_{\theta}\left(\rho_{1}\right)
$$

where the barred quantities correspond to the particular solution of $\left(6^{6} .6\right)$,

The time parameters $C_{1}, C_{2}$ of the elastic domain appearing in $(6.17 \mathrm{~b})$ are no longer given by (5.7) but must be redetermined from (3.1) with the additional boundary condition $\left(a_{\theta}-\sigma_{z}\right)_{r=\rho}=2 k_{0}$ giving

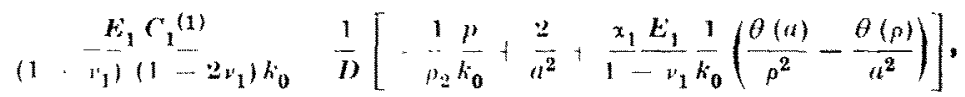

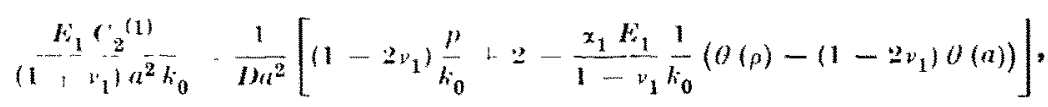

where $D=\frac{1}{p^{2}}+\frac{1-2^{2} t}{a^{2}}$.

With $C_{1}^{(1)}$ thus determined it is approprinte to record here the relation between $\gamma^{\prime}$ and $\Phi_{1}$ for Stage I, which may be obtained from (5.6) through (5.7b) and (6.19a):

$$
\begin{aligned}
& \frac{p}{k_{0}}\left[1+v_{1}-\frac{2\left(1-v_{1}^{2}\right)}{b_{p}^{2}}-\frac{k_{1}^{5}}{E_{42}}\left(1, v_{2} \cdot \frac{2\left(1-v_{2}^{2}\right)}{b^{2} a^{2}-1}\right)\right]
\end{aligned}
$$

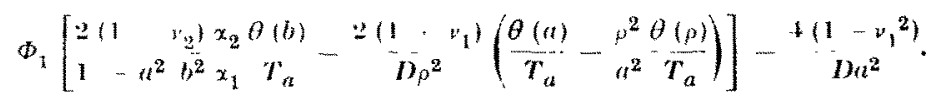

Equating $(6.17 a)$ and $(6.18 b)$, there results two equations in the unknowns $\Phi_{1}, \rho_{1}$, and $\rho$ which may be best solved numerically for various shoiees of $\rho$. With $\phi_{1}$ and $\rho_{1}$ determinad, all of the coefficients $C_{1}, C_{2}, A_{1}, A_{2}, D_{3}$, given by $(6.19 \mathrm{a}),(6.19 \mathrm{~b}),(6.17 \mathrm{a})$ and $(6.14)$, respectively, may be evaluated, and the solution for this stage of the problem is complete.

\section{Stages $I I$ and $I I I$}

Due to the nature of the problem and the yield condition (6.1), when the elastic-plastic boundary $\rho$ reaches the outer radius $r=a$, the interface boundary $p_{1}$ continues to propagate and the cylinder is still capable of ateepting more loak, sven in the absence of workhardening.

Since zone II now extends to the interfuce $r=a$, the relation $(6.20)$ ceases to be valid and a new relation between $p$ and $\Phi_{1}$ must be obtained. Since the cladding is still assumed to be elastic, (5.5) with $i=2$ remains valit and is sel equal $b$ (0.9) at $r=a k$.

A second relation between $p$ and $\Phi_{1}$ may be found by substitution of (6.18) (which remains valid in stage $I$, since the form of the solution in zone 1 has not changed) into the boundary condition $\pi_{r}(a)=-p$. The two new relafinns hetween $p$ and $\phi_{1}$ may then be solved simultaneously for the two unknowns, Throughout Stage II, in the absence of an elastic-plastic boundary during Stage $\mathrm{H}, \rho_{1}$ may be treated as the independent variable. 
The elastio phatie cylinder subjected to radially distributed heat source

The description of the plastie deformation provided hy stage II continues to be valid, untul the state of stress at the outer radius $r=a$ of the cylinder. represented during Stage II by a point moving in regime $B C$ of Fig. 1 , reaches the singular regime $B$, at which time the solution enters Stage III. As the states of stress at sucressive points in zone II of the cylinder enter regime $B$, a second interface $\rho_{2}$ is formed which moves inward from $r=a$ and forms a plastic domain III $\left(\rho_{2}=r \leqq a\right)$, corresponding to state's of stress lying in regime $B$.

The differential equation governing the radial stress $\sigma_{r}$ (as well as $\sigma_{z}$, since $\sigma_{z}=\sigma_{z}$ ) in zone III, derived in a manner similar to $(6,6)$ for zone $1 I$, is given by

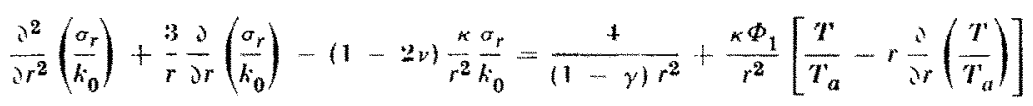

for a workhardening material. The tangential stress $\sigma_{\theta}$ is then obtained through the equilibrium equation (2.1) and the displacement $"$ through the compressibility condition (4.6a). For a nonhardening material the stress field is given by $(4,17)$. The method of determining the coeflicients appearing in the solution is similar to that if stage $I$ with $\rho$ replaxed by $\rho_{2}$ and $\gamma_{1}$ and $\beta_{1}$. in ( $6.17 \mathrm{a})$ replaced respectively by

$$
\gamma_{3}=\sigma_{r}\left(p_{2}\right)-\sigma_{r}\left(p_{2}\right) . \quad \beta_{3} \cdots \sigma_{\theta}\left(\rho_{2}\right)-\sigma_{\theta}\left(\rho_{2}\right)
$$

where $\sigma_{r}\left(\rho_{2}\right)$ and $\sigma_{\theta}\left(\rho_{2}\right)$ in (6.22) reler to the components of tress m zone 111 found from (6.21) (or $(4.17)$ ).

Subsequent to the determination of a new relation between $p$ and $Q_{0}$ for Stage 111 , and the redeternination of the time fonetions in the stress and displacenent fields, it is found that the separation boundaries $\rho_{1}$ and $\rho_{2}$ approach each other monotonically but do not meet : eakh approanhing a limit value as the temperature is imdefinitely increased. This is reminisent of the results given by WiLhor (19.58) for a ring in the state of plane stress, subjected to a temperature gradient, and by Cowpen (1958) for a hollow sphere also in the presence of a temperature gradient.

This completes the solution of Stige III of the problem which remuins valid until yield ocenr in the cladding. It may be noted here that, once the cladding has yielded, a fourth stage of the deformation begins, since the interaction of the three boundaries $\rho_{1}, \rho_{2}$ and $\rho_{3}$ (the elastic-plastic. boundary in the cladding) must be considered.

\section{Discenston - Numerical Resuits}

In the preceding Sections the character of the initial yield and the subsequent deformation was established for a class of radially symmetric temperature distributions $(1 \leqq m \leqq 2)$, chosen to include (5.2) which was due to a Gaussian heat source, distributed throughout the region. The expressions in Section 6 are written in general terms to include any temperature distribution in this class. In order to gain an insight into the problem, it is desirable to consider a detailed example for one case. Since the expressions in Section 6 become involved and intricate, when $(5.2)$ is inserted, for the sake of simplicity we allow the parameter $\mu^{2}$ to approach zero. The temperature distribution $(5.2)$ then assumes the simple form

$$
T=T_{a}+\frac{Q_{0} a^{2}}{4 K_{1}}\left[1-\left(\frac{r}{a}\right)^{2}\right]
$$

and $\frac{\theta(r)}{T_{a}}$ becomes

where

$$
\frac{\theta(r)}{T_{a}}=\frac{1}{2}\left\{1+\delta\left\{1-\frac{1}{2}\left(\frac{r}{a}\right)^{2}\right]\right\}
$$

$$
\delta=\frac{a^{2}}{4 K_{1} h}
$$


and by $(5.11 \mathrm{~b})$

$$
h=-\frac{T_{a}}{Q_{0}}=\frac{a^{2} \log b^{\prime} a}{2\left(1-T_{b} T_{a}^{-}\right) \bar{K}_{2}} .
$$

Comparison plots of the functions specified by (5.2) and (7.1) as well as the integrals which appear in the solution reveal a qualitative similarity between the two temperature distributions, which together with the fact that both produce initial yield of the same character $(m \ldots 1$, so that the results in Table 1 are applicable) justifies the use of (7.1) in place of the more realistic expression (5.2). The resulting equations are thus considerably simplified.

We now proceed to obtain explicit results for a workhardening material obeying (2.5) subjected to a uniformly distributed heat source $\left(\mu^{2}=0\right)$ which gives rise to the temperature field (7.1). In each expression the nonhardening material may be obtained by setting $\gamma=\kappa=0$.

For the values of the parameters $(5.16 a)$ selected in Section 5 we continue the example and obtain explicit expressions using the alternative temperature field (7.1) for the quantities discussed in Section 6. In zone I for all stages of the problem the state of stress and the displacement continue to be given by (4.18) and (6.16) with $D_{3}$ and $k$ becoming through $(6.11)$ and (6.15), respectively:

$$
\left.\begin{array}{r}
\frac{1-2 \nu_{1} \frac{D_{3}}{2}=}{k_{0}}=1-\frac{\Phi_{1}}{2}\left(1+\delta\left[1-\left(\frac{\rho_{1}}{a}\right)^{2}-\left[\frac{5}{2}+\gamma\left(1+v_{1}\right)\right]\right]\right\} \\
\left.\frac{k}{k_{0}}=1-\frac{\Phi_{1} \delta \kappa}{2}\left(\left(\frac{r}{a}\right)^{2}-\left(\frac{\rho_{1}}{a}\right)^{2}\left[\frac{5}{2}+\gamma\left(1+v_{1}\right)\right]\right]\right\}
\end{array}\right\}
$$

In zone II for all stages the stresses may be found by allowing $\mu^{2}$ to approach zero in (6.8) or more easily from (6.6). the right-hand side of which upon substitution of (7.1) becomes

where

$$
b_{3}+b_{4}\left(\frac{r}{a}\right)^{2}
$$

$$
\left.\begin{array}{l}
b_{3}=\frac{1}{1+\gamma}\left[2-\Phi_{1}(1+\delta)\right] \\
b_{4}=\Phi_{1} \delta\left[5-\left(3-2 \nu_{1}\right) \kappa\right]
\end{array}\right\}
$$

and consequently the stress field in zone II is

$$
\left.\begin{array}{l}
\frac{\sigma_{y}}{k_{0}}=\frac{A_{5}}{r^{s_{5}}}+\frac{A_{6}}{r^{s_{6}}}+A_{7}+A_{8}\left(\frac{r}{a}\right)^{2} \\
\frac{\sigma_{\theta}}{k_{0}}=\left(1-s_{5}\right) \frac{A_{5}}{r^{s_{5}}}+\left(1-s_{6}\right) \frac{A_{6}}{r^{s_{6}}}+A_{7}+3 A_{8}\left(\frac{r}{a}\right)^{2}, \\
\frac{\sigma_{z}}{k_{0}}=\frac{\sigma_{\theta}}{k_{0}}-\frac{2 k}{k_{0}}
\end{array}\right\}
$$

where

$$
A_{7}=\frac{b_{3}}{b_{2}}, \quad A_{8}=\frac{h_{4}}{8 b_{1}+b_{2}}
$$

and $k$ is given by $(6.4)$. 
The elastic-plastic cylinder subjected to radially distributed heat source

By (7.5) and (7.9), $A_{5}$ and $A_{\mathfrak{6}}$ may be found through (6.18) to be

$$
\left.\begin{array}{l}
A_{5}=\frac{-1}{s_{5}-s_{6}} \frac{\rho_{1}^{s_{5}+2}}{a^{2}}\left\{\frac{s_{6}}{1-2 \Phi_{1} \delta}\left[5 / 2+\gamma\left(1+\nu_{1}\right)\right]-\left(s_{6}+2\right) A_{8}\right\}, \\
A_{6}=\frac{1}{s_{5}-s_{6}} \frac{\rho_{1}{ }^{s_{6}+2}}{a^{2}}\left\{\frac{s_{5} \Phi_{1} \delta}{1-2 \nu_{1}}\left[5 / 2+\gamma\left(1+v_{1}\right)\right]-\left(s_{5}+2\right) A_{8}\right\},
\end{array}\right\}
$$

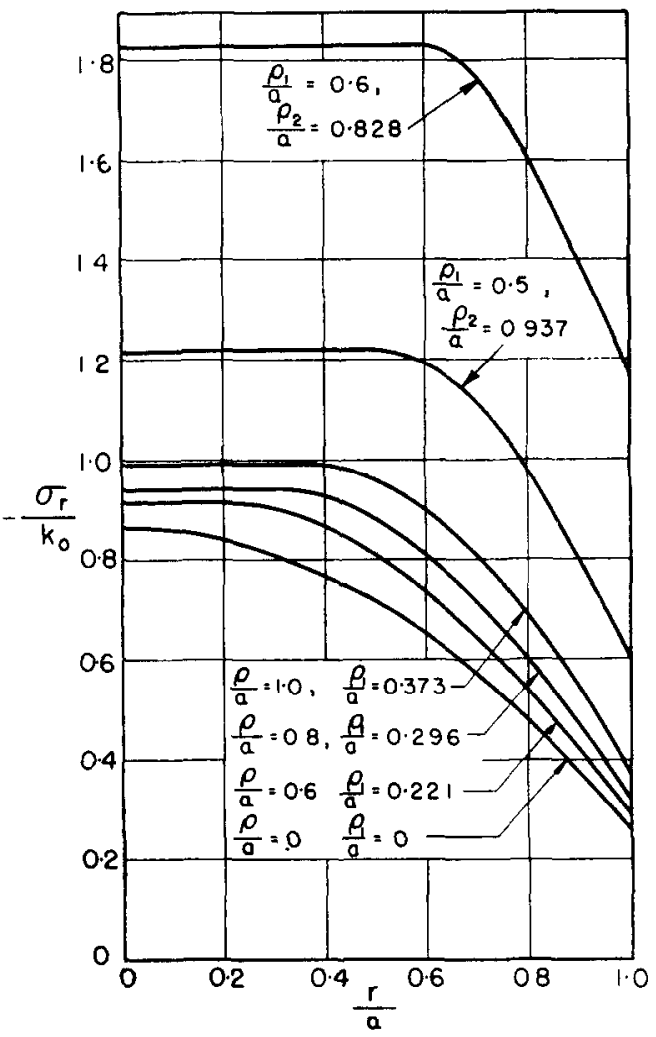

FI(. 3. Distribution of radial stress $(\gamma=0)$.

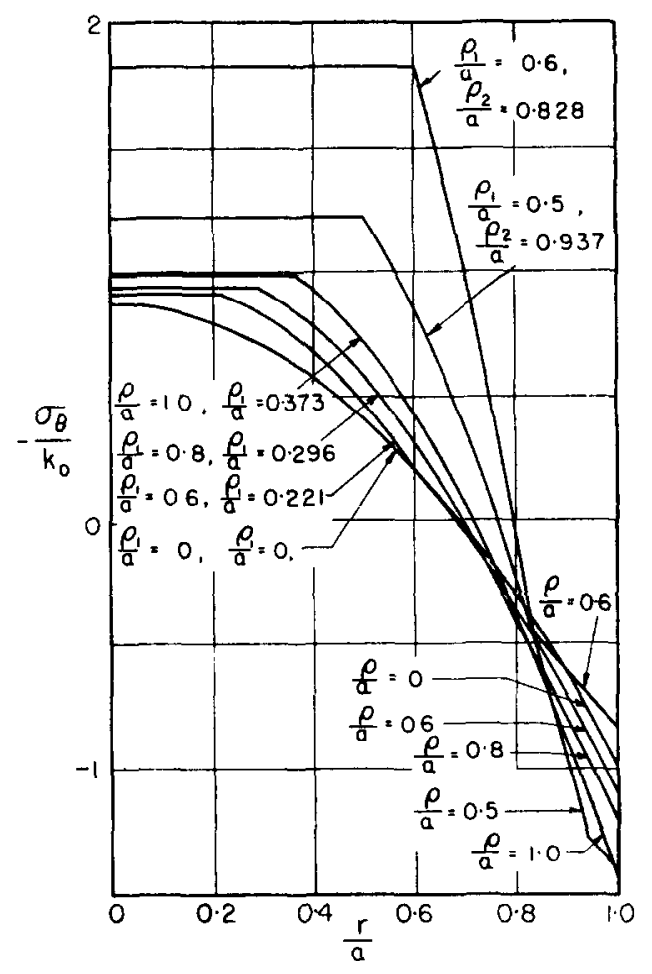

FIs. 4. I)istribution of tangential stress $(\gamma=0)$.

Fquating these values of $A_{5}$ and $A_{6}$ with those given by (6.17a) with subsequent elimination of $\rho_{1}$ between the two resulting equations leads to the following relation :

$$
\left.\begin{array}{rl}
\left(\frac{\rho}{a}\right)^{2\left(s_{6}-s_{6}\right)} & \left\{\frac{\gamma_{1}\left(1-s_{6}\right)-\beta_{1}}{-s_{6} \Phi_{1} \delta\left[5,2+\gamma\left(1+\nu_{1}\right)\right]+\left(2+s_{6}\right) A_{8}}\right\}^{2+s_{6}} \\
= & \left\{\frac{\gamma_{1}\left(1-s_{6}\right)+\beta_{1}}{1-2 v_{1}}\right]^{2+s_{6}},
\end{array}\right\}
$$

which indicates the complex nature of the interaction between $p, \Phi_{1}$ and $\rho$ even for the simple distribution (7.1). Equation (7.11) may be solved simultaneously with (6.20) to determine $p$ and $\Phi_{1}$ in terms of $\rho$. 
The values $p^{* *}, Q_{0}{ }^{* *}$ for which the cross-section becomes fully plastic, signifying the start of Stage II, may also be obtained from (7.11) and (6.20) by setting $\rho^{\prime} a-1$. During stage II of the problem (7.5) continues to hold in zone I and (7.10) in zone II. Only the means of obtaming relations between $p$ and $\Phi_{1}$. discussed in Section 6 , differs from Stage I. Stage II ends when $\sigma_{r}=\sigma_{z}$ at $r-a$ signifying that the state of stress has entered a singular regime $(B)$, constituting the start of Stage III. The procedure used in the solution of Stage III parallels Stage I and for economy of space will not be included here.

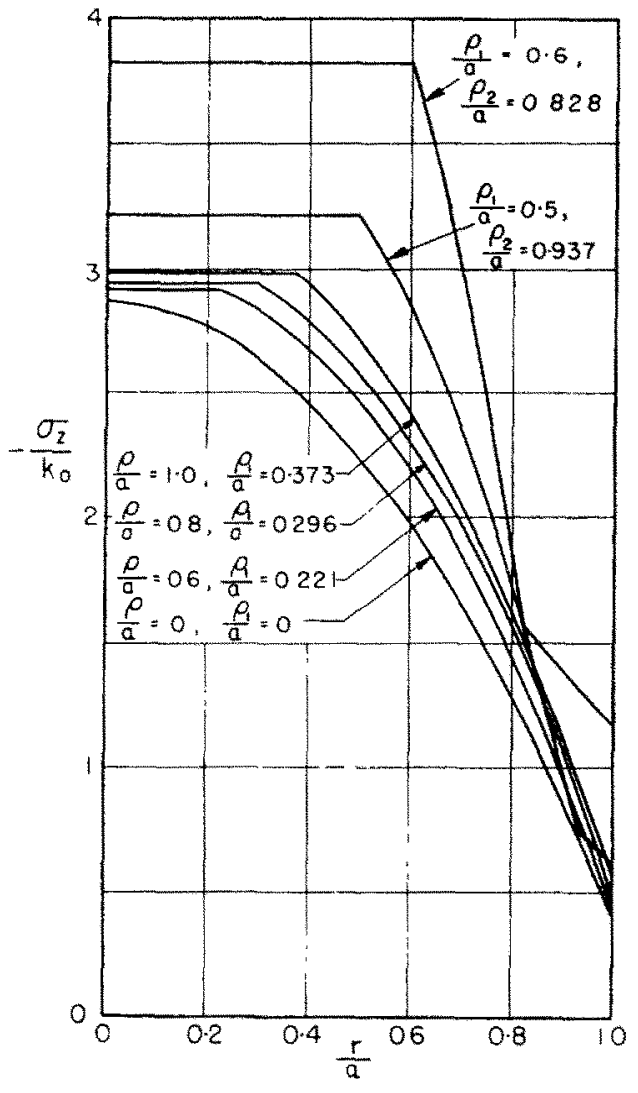

Frg. 5. Distribution of axial stresc $(y$ (b).

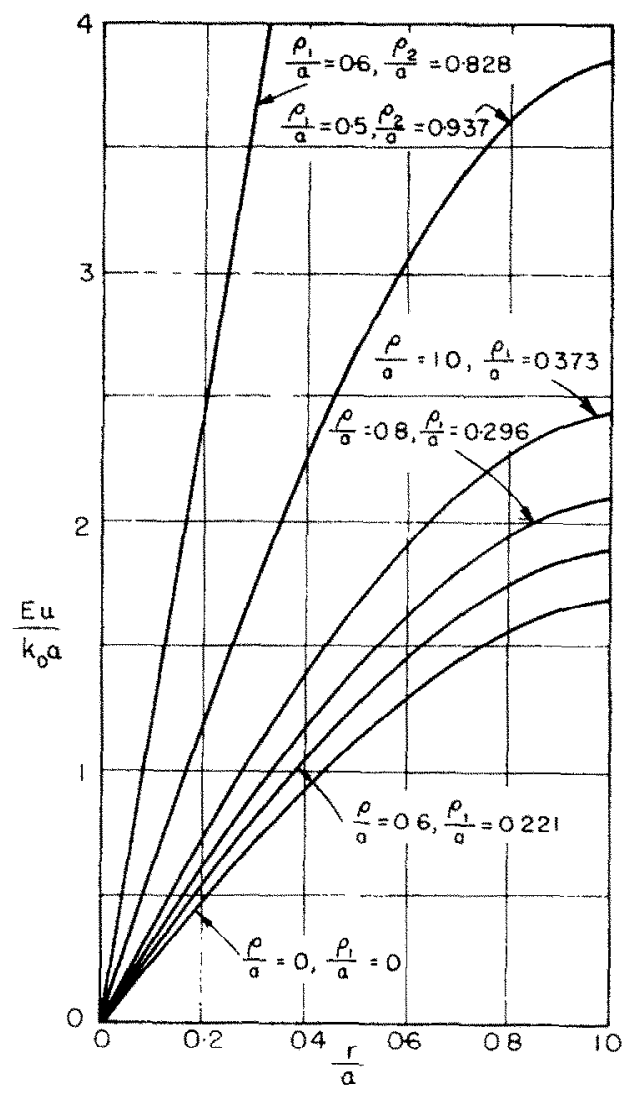

Fia. A. Ratio of radial displacement to (ylunder rudius $(\gamma=0-3)$.

For a nonhardening material $(\gamma=0)$ the stress distributions and displacements for various positions of $\rho_{1}$ are shown in Figs. $3-6$, and the projection of the loading paths on a plane perpendicular to the axis of the yield surface is shown in Fig. 2.

To illustrate the effect of workhardening, a very drastic value 0.3 has been selected for $\gamma$; this corresponds roughly to the value of $\gamma$ for uranium in compression (GIRINSkY and DikNes 1956, p. 58).

Although not included in the Figures, it is found that at the completion of Stage I the greatest difference between the hardening and nonhardening cases occurs in the axial component of stress $(7+2$ per cent at $r=0)$; the plane components differ 


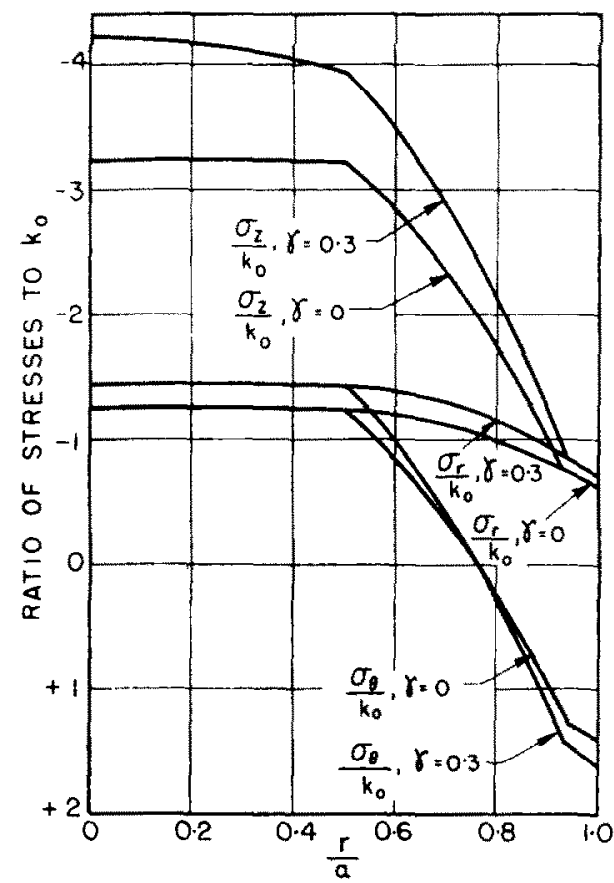

Fig. 7 . Comparison of the three components of stress for hardening $(\gamma=0 \cdot 3)$ and nonhardening $(\gamma=0)$ materials when $\frac{p_{1}}{a}=0.5$ during Stage III.

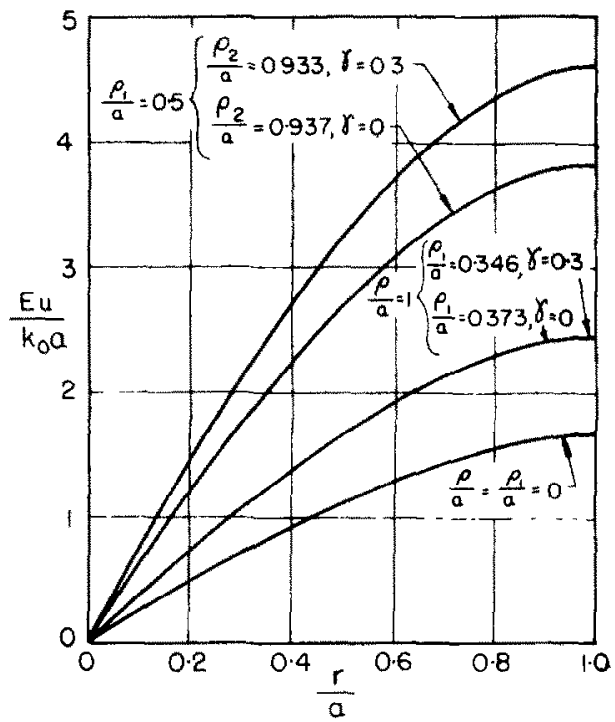

Fic. 8. Comparison of the ratio of radal displacement to cylinder radius for hardening $(\gamma=0 \cdot 3)$ and nonhardening $(\gamma \cdots 0)$ materials.

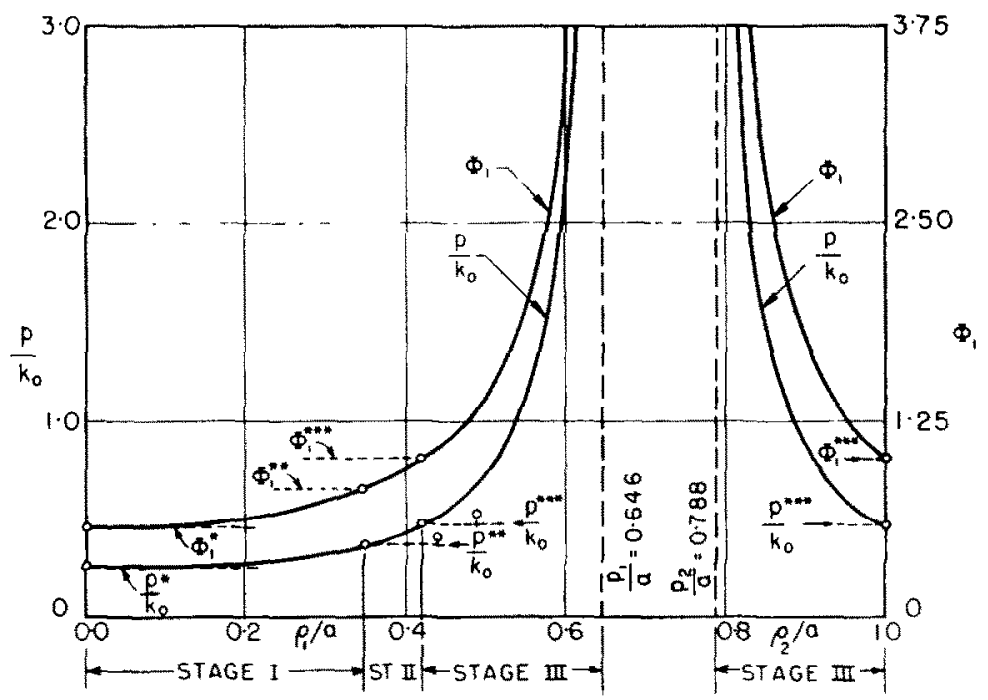

FIG. 9. Load parameters for various positions of the boundaries $\rho_{1}$, and $\rho_{2}$, showing values at initial yield $\left(p^{*}, Q_{0}{ }^{*}\right)$, at the start of Stage II $\left(p^{* *}, Q_{0}{ }^{*}\right)$, and Stage III $\left(p^{* * *}, Q_{0}^{* *}\right)$, as well as the asymptotic values of $\rho_{1}$ and $\rho_{2}$. 
by less than 2 per cent, while the radial displacements (Fig. 8) are indistinguishable. Iarger differences occur during Stages II and III and in Figs. 7 and 8 the components of stress and displacement. respeclively, for $\rho_{1}=0.5$ of stage III are compared. The character of the yield stress in simple shear for two values of $\rho_{1}$ is shown in Fig. 10. The load parameters for the workhardening case are shown in Fig. 9 as a function of $\rho_{1}$ and $\rho_{2}$. The boundaries $\rho_{1}$ and $\rho_{2}$ for a workhardening material are found to lag behind the nonhardening values for a given value of the load parameters $p$ and $\Phi_{1}$.

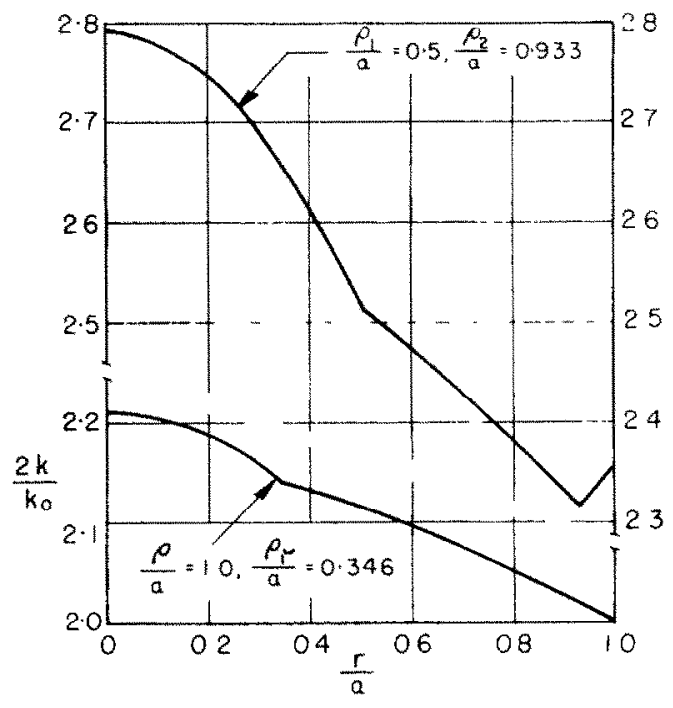

Fre. 10. Vind limit in shexpr at the eompletion of stage I and when $\frac{p_{1}}{a}=0.5$ shortly after the start of stimge 111.

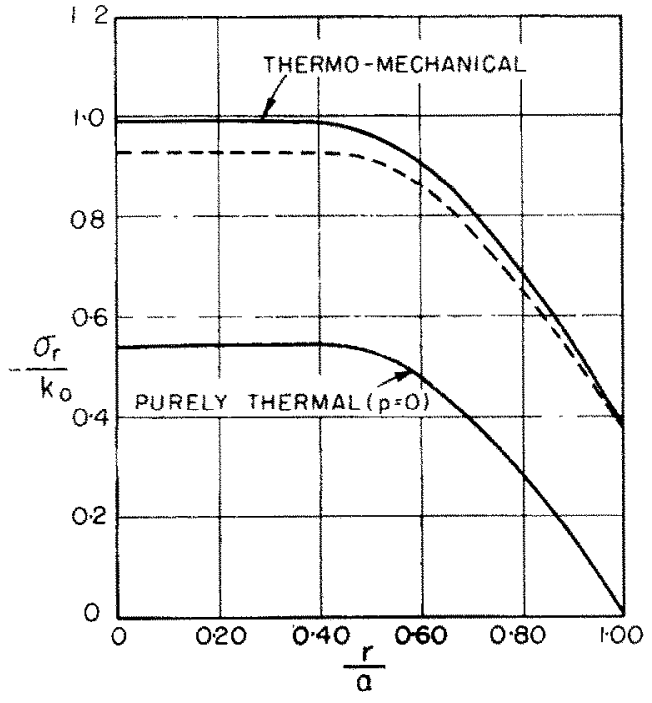

HIG. 11. Comparison of the thermo-mechanieal solution at the completion of Stage I for the state of plane strain with that obtained by superposition of the purely thermal solution for the state of plane strain and hydrostatic pressure (shown by dashed lines) for $\gamma=0$.

Finally it may be noted that the effect of adding $\sigma_{r}=-p$ to the purely thermal problem $(p-0)$ is not a matter of the simple super-position of a hydrostatic pressure. In fact the plane strain conditions prevent this so that the elastic-plastic deformation depends to a great extent on the relation between $\Phi_{1}$ and $p$. To illustrate this, a comparison of $\sigma_{r}$ at the completion of Stage I for the thermomechanical problem, considered in this paper, with the result obtained by superimposing a hydrostatic pressure upon the purely thermal solution* is shown in Fig. 11.

\section{REFERFNCES}

\begin{tabular}{|c|c|c|}
\hline BHANI, D, R. & $\begin{array}{l}19.50 \\
19.5 \%\end{array}$ & $\begin{array}{l}\text { J. Mech. Phys. Solids 4, } 200 \text {. } \\
\text { Proc. 9th Int, Congr. Ippl. Mech. 8, } 45 .\end{array}$ \\
\hline ( OWPFR, $\mathrm{C}, \mathrm{R}$. & 195 & $\begin{array}{l}\text { Terh. Report No. } 9 \text {, Nonr } 562(30) \text {, Wivision of Engsineering, } \\
\text { Brown Iniversity. }\end{array}$ \\
\hline
\end{tabular}

- It is easily verined bo $(514)$ that the chameter of intial yield is unchanged for the purely thermal problen 


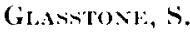

(GIBISKY, I). HI. and I) II:Sis. (;. .J.

HII,L, IR.

HoI)(;F, l'. G.

ForTter, IV. 'T.

Pankes, II.

pragier. W.

Thompsox, $A$. S. and Rungrers, O. E.

'TIMOSHFNko, $\$$. and Goontt:k, J. I.

VEITF:R, J. II,

Wener, J. H. and Heddestox. J. $\mathrm{V}$. WilhoIT, J. ('.
19.55

1956

$195 i$

1956

19.53

1954

1958

1936

1951

1956

19.59

1958
Principles of Vuclear Reactor Engineering (Van Nostrand. New York).

Nuclear Fuels (Van Nostrand, Princeton).

Mathematical Theory of Plasticity (Clarendon Press, Oxford). J. Rat. Mech. Anal. 5. 917.

Biezeno Anniversary Volume on Applied Mechanics, p. $2: 32$ (Hatarem, Holland).

Proc. 2nd C..s. Nat. Congr. Appl. Mech., 301.

Proc. Ken. Ned. Ahad. v. Wetensch. B61, 176.

Thermal Pozer from Nuclear Reactors (.John Wiley. New York).

Theory of Elasticity (McGraw-Hill, New York). J. 1ppl. Hech. 23, 395 .

J. Appl. Mech. 26, 31 .

Proc. 3rd I.S. Nat. Congr. 1ppl. Mech., 693. 